\title{
Wiener Filtering for Passive Linear Quantum Systems
}

\author{
V. Ugrinovskii
}

M. R. James

\begin{abstract}
This paper considers a version of the Wiener filtering problem for equalization of passive quantum linear quantum systems. We demonstrate that taking into consideration the quantum nature of the signals involved leads to features typically not encountered in classical equalization problems. Most significantly, finding a mean-square optimal quantum equalizing filter amounts to solving a nonconvex constrained optimization problem. We discuss two approaches to solving this problem, both involving a relaxation of the constraint. In both cases, unlike classical equalization, there is a threshold on the variance of the noise below which an improvement of the mean-square error cannot be guaranteed.
\end{abstract}

\section{INTRODUCTION}

The task of transferring quantum information differs significantly from its classical (non-quantum) counterpart, since the laws of quantum mechanics limit the accuracy of information transfer through quantum channels. Specifically, the signal-to-noise ratio of possible quantum measurements on the transmission line is limited [4], reflecting the well known fact that a quantum state cannot be cloned at the remote location. This motivates a great interest in developing systematic methodologies for the design of optimally performing quantum communication systems.

In the classical communication theory, optimization plays an instrumental role in balancing various trade-offs in the design of classical communication systems. The most celebrated example of using optimization in signal processing are due to N. Wiener [15] who developed a general method for reducing the effects of noise and channel distortion through minimization of the mean square error (MSE) between the signal and its estimate over a class of linear filters. This paper highlights conceptual challenges that arise when the Wiener optimization paradigm is applied in the derivation of coherent quantum filters, i.e., filters which themselves are quantum systems. To be concrete, we restrict attention to one type of the coherent filtering problem concerned with equalizing distortions of quantum signals transmitted via a quantum communication channel. Owing to the analogy with classical channel equalization, we call this problem the quantum equalization problem. The paper shows that the requirement for the filter to be physically realizable translates

This work was supported by the Australian Research Council and the ARC Centre for Quantum Computation and Communication Technology.

V. Ugrinovskii is with the School of Engineering and Information Technology, University of New South Wales at the Australian Defence Force Academy, Canberra, ACT 2600, Australia, V.ougrinovskieadfa.edu.au

M. R. James are with the ARC Centre for Quantum Computation and Communication Technology, Research School of Engineering, The Australian national University, Canberra, ACT 2601, Australia, matthew. james@anu. edu.au. into additional constraints which render the problem of optimizing the mean square of the equalization error nonconvex.

The paper is centered around the so-called passive quantum equalizers. Mathematically, dynamics of a passive quantum system in the Heisenberg picture are described by complex quantum stochastic differential equations expressed in terms of annihilation operators only [7]. Such systems are simple to implement experimentally by cascading conventional quantum optics components such as beam splitters and optical cavities [11]. Furthermore, in a general quantum system, passivity ensures that the system dissipates energy in the input. A striking observation that emerges from our analysis is that passivity appears to be a rather restrictive property in the context of equalization, in that an optimal passive coherent equalizer is not always able to improve the MSE. It turns out that the achievable improvement depends on the variance of the quantum noise in the filter input signal. We give examples which reveal a threshold on this variance above which the optimal passive coherent equalizer delivers an improved MSE.

The paper is organized as follows. In the next section we present the basics of passive linear quantum systems. The quantum passive equalization problem is posed in Section III A relaxation of the problem is proposed in Section IV Next, in Section $\mathrm{V}$, the problem is particularized to demonstrate the dependency between the power spectrum density of the equalization error and the variance of the system noise. Two examples of the quantum coherent filter design are presented in that section, reflecting two approaches to optimization of the equalization error, the first one is via direct optimization of the power spectrum density, and the second one is using the Wiener-Hopf factorization technique [8]. Finally, concluding remarks are given in Section VI.

Notation: For an operator $a$ in a Hilbert space $\mathfrak{H}, a^{*}$ denotes the Hermitian adjoint operator, and if $a$ is a complex number, $a^{*}$ is its complex conjugate. Let $a=\left(a_{1}, \ldots, a_{n}\right)$ be a column vector comprised of $n$ operators (i.e., $a$ is an operator $\left.\mathfrak{H} \rightarrow \mathfrak{H}^{n}\right)$; then $a^{\#}=\left(a_{1}^{*}, \ldots, a_{n}^{*}\right), a^{T}=$ $\left(a_{1}^{T} \ldots a_{n}^{T}\right)$ (i.e, the row of operators), and $a^{\dagger}=\left(a^{\#}\right)^{T}$. The notation $\operatorname{col}(a, b)$ denotes the column vector obtained by concatenating vectors $a$ and $b$. For a complex matrix $A=\left(A_{i j}\right), A^{\#}, A^{T}, A^{\dagger}$ denote, respectively, the matrix of complex conjugates $\left(A_{i j}^{*}\right)$, the transpose matrix and the Hermitian adjoint matrix. $[\cdot, \cdot]$ denotes the commutator of two operators in $\mathfrak{H} \cdot \operatorname{tr}[\cdot]$ denotes the trace of a matrix. $I$ is the identity matrix. The quantum expectation of an operator $V$ with respect to a state $\rho$, is denoted $\langle V\rangle=\operatorname{tr}[\rho V][12]$. 


\section{OPEN LINEAR PASSIVE QUANTUM SYSTEMS}

An open quantum annihilation-only system represents a linear system

$$
\begin{aligned}
& \dot{\mathbf{a}}=A \mathbf{a}+B \mathbf{u}, \quad \mathbf{a}\left(t_{0}\right)=\mathbf{a}, \\
& \mathbf{y}=C \mathbf{a}+D \mathbf{u} ;
\end{aligned}
$$

where $A, B, C, D$ are complex $m \times m, m \times n, n \times m$, $n \times n$ matrices, and $u$ is a (column) vector of $n$ quantum input processes. The input is assumed to be of the form

$$
\mathbf{u}(t)=\mathbf{u}_{0}(t)+\mathbf{b}(t),
$$

where $\mathbf{b}$ is a (column) vector of $n$ quantum noise processes, $\mathbf{b}=\left(\mathbf{b}_{1}, \ldots, \mathbf{b}_{n}\right)$, and $\mathbf{u}_{0}(t)$ is an adapted process [5]. The noise processes can be represented as annihilation operators on an appropriate Fock space [5], but from the system theory viewpoint they can be treated as quantum Gaussian white noise processes with zero mean, and the covariance

$$
\left\langle\left[\begin{array}{c}
\mathbf{b}(t) \\
\mathbf{b}^{\#}(t)
\end{array}\right]\left[\begin{array}{c}
\mathbf{b}^{\dagger}\left(t^{\prime}\right) \\
\mathbf{b}^{T}\left(t^{\prime}\right)
\end{array}\right]\right\rangle=\left[\begin{array}{cc}
I+\Sigma_{\mathrm{b}}^{T} \Pi_{\mathrm{b}} \\
\Pi_{\mathrm{b}}^{\dagger} & \Sigma_{\mathrm{b}}
\end{array}\right] \delta\left(t-t^{\prime}\right),
$$

where $\Sigma_{\mathrm{b}}, \Pi_{\mathrm{b}}$ are complex matrices with the properties that $\Sigma_{\mathrm{b}}=\Sigma_{\mathrm{b}}^{\dagger}, \Pi_{\mathrm{b}}^{T}=\Pi_{\mathrm{b}}$. Along with their adjoint (creation) operators $\mathbf{b}_{j}^{*}(t)$, the noise operators satisfy canonical commutation relations $\left[\mathbf{b}_{j}(t), \mathbf{b}_{k}^{*}\left(t^{\prime}\right)\right]=\delta_{j k} \delta\left(t-t^{\prime}\right)$, $\left[\mathbf{b}_{j}(t), \mathbf{b}_{k}\left(t^{\prime}\right)\right]=0$. Here, $\delta_{j k}=0$ when $j \neq k$, and is the identity operator when $j=k ; \delta\left(t-t^{\prime}\right)$ is the $\delta$-function. The column vector $\mathbf{a}(t)=\left(\mathbf{a}_{1}(t), \ldots, \mathbf{a}_{m}(t)\right)$ represents the system modes and consists of annihilation operators on a certain Hilbert space $\mathfrak{H}$. A discussion about open linear quantum systems can be found in [7], [2], [6]. From now on, it will be assumed that the pair $(A, B)$ is controllable.

For a system of the form (1) to correspond to quantum physical dynamics, it must preserve the canonical commutation relations during its evolution [13], [6]. According to [9], for the system (1) this is guaranteed if and only if there exists a Hermitian complex matrix $\Theta$ such that

$$
A \Theta+\Theta A^{\dagger}+B B^{\dagger}=0, \quad B=-\Theta C^{\dagger}, \quad D=I .
$$

Without loss of generality we will assume from now on that the conditions (4) are satisfied for the systems under consideration with $\Theta=I$; this can always be achieved by an appropriate choice of coordinates [9]. Furthermore, we will assume that the matrix $A$ is Hurwitz.

From (1), the output of the system can be represented as

$$
\begin{aligned}
\mathbf{y}(t)= & C e^{A\left(t-t_{0}\right)} \mathbf{a} \\
& +\int_{t_{0}}^{t} g(t-\tau) \mathbf{u}_{0}(\tau) d \tau+\int_{t_{0}}^{t} g(t-\tau) \mathbf{b}(\tau) d \tau
\end{aligned}
$$

Here we introduced the notation for the impulse response, associated with the system [16],

$$
g(t)= \begin{cases}C e^{A t} B+\delta(t) I, & t \geq 0, \\ 0, & t<0 .\end{cases}
$$

Let us introduce the transfer function of the system (1),

$$
G(s)=C(s I-A)^{-1} B+I .
$$

Since $B=-C^{\dagger}$, the transfer function $G(s)$ is square. This observation holds for all passive systems considered henceforth. Furthermore, if follows from the properties of the physical realizability [13] that for the passive system (1),

$$
G(s)\left[G\left(-s^{*}\right)\right]^{\dagger}=I .
$$

In the sequel we will be interested in stationary behaviours of the systems under consideration. Since the matrix $A$ is assumed to be stable and assuming that $\mathbf{u}_{0}(t)$ is stationary, the stationary component of the system output is obtained from (5) by letting $t_{0} \rightarrow-\infty$ :

$$
\mathbf{y}(t)=\int_{-\infty}^{+\infty} g(t-\tau) \mathbf{u}_{0}(\tau) d \tau+\int_{-\infty}^{+\infty} g(t-\tau) \mathbf{b}(\tau) d \tau(8)
$$

Also, for convenience the upper limit of integration has been changed to $+\infty$ since $g(t)$ is causal.

Consider the correlation function of stationary quantum operator processes $\mathbf{x}_{j}(t), \mathbf{x}_{k}(t)$ associated with the system,

$$
R_{\mathbf{x}_{j}, \mathbf{x}_{k}}(t)=\left\langle\left(\mathbf{x}_{\mathbf{j}}(0)-\left\langle\mathbf{x}_{\mathbf{j}}(0)\right\rangle\right)\left(\mathbf{x}_{\mathbf{k}}{ }^{*}(t)-\left\langle\mathbf{x}_{\mathbf{k}}{ }^{*}(t)\right\rangle\right)\right\rangle .
$$

The corresponding power spectrum density is then

$$
P_{\mathbf{x}_{\mathbf{j}}, \mathbf{x}_{\mathbf{k}}}(i \omega)=\int_{-\infty}^{+\infty} e^{-i \omega t} R_{\mathbf{x}_{j}, \mathbf{x}_{k}}(t) d t .
$$

The Fourier transform is understood in the sense of tempered distributions when $R_{\mathbf{x}_{j}, \mathbf{x}_{k}}$ is not integrable. Also, consider the extension of $P_{\mathbf{x}_{\mathbf{j}}, \mathbf{x}_{\mathbf{k}}}(i \omega)$ to the complex plane, given by the bilateral Laplace transform of $R_{\mathbf{x}_{j}, \mathbf{x}_{k}}$,

$$
P_{\mathbf{x}_{\mathbf{j}}, \mathbf{x}_{\mathbf{k}}}(s)=\int_{-\infty}^{+\infty} e^{-s t} R_{\mathbf{x}_{j}, \mathbf{x}_{k}}(t) d t
$$

Often, $P_{\mathbf{x}_{\mathbf{j}}, \mathbf{x}_{\mathbf{k}}}(s)$ is also referred to as the power spectrum density function [8], although in general it may not be real. Since the matrix $A$ is Hurwitz, $P_{\mathbf{x}_{\mathbf{j}}, \mathbf{x}_{\mathbf{k}}}(s)$ is well defined on $s=i \omega$ and $\left.P_{\mathbf{x}_{\mathbf{j}}, \mathbf{x}_{\mathbf{k}}}(s)\right|_{s=i \omega}=P_{\mathbf{x}_{\mathbf{j}}, \mathbf{x}_{\mathbf{k}}}(i \omega)$, where the expression on the left-hand side refers to the power-spectrum density defined in (10), and the expression on right-hand side is defined in (9). It is easy to obtain that the power spectrum density matrix of the output $\mathbf{y}(t), P_{\mathbf{y}, \mathbf{y}}(s)=\left(P_{\mathbf{y}_{j}, \mathbf{y}_{k}}(s)\right)$ is related to the power spectrum density matrix of the noise $\mathbf{b}$, $P_{\mathbf{b}, \mathbf{b}}(s)=\left(P_{\mathbf{b}_{j}, \mathbf{b}_{k}}(s)\right)$, in the standard manner:

$$
P_{\mathbf{y}, \mathbf{y}}(s)=G(s) P_{\mathbf{b}, \mathbf{b}}(i \omega)\left[G\left(-s^{*}\right)\right]^{\dagger} .
$$

\section{EQUALIZATION PROBLEM FOR ANNIHILATION-ONLY COMMUNICATION SYSTEMS}

In this section, a general equalization scheme for a passive communication system is outlined.

Consider a system in Fig. 1 consisting of a quantum channel and an equalizer. The input signal $u$ plays the role of a message signal to be transmitted through the channel, of the form

$$
u(t)=u_{0}(t)+b(t),
$$

and $w$ denotes the vector comprised of additional quantum noises. It includes the noise inputs that are necessarily present in the physically realizable system $G(s)$ [6], [14], as well as noises introduced by measurement devices. In 


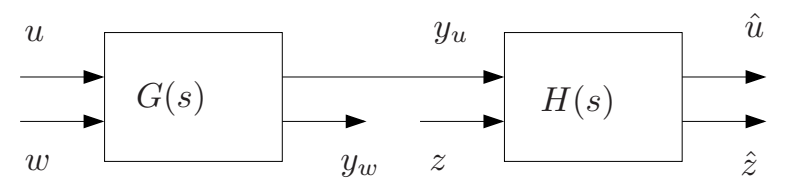

Fig. 1. A general quantum communication system. The transfer function $G(s)$ represents the channel, and $H(s)$ represents an equalizing filter.

terms of the notation adopted in the previous section, we have $\mathbf{u}_{0}=\operatorname{col}\left(u_{0}, 0\right)$, and $\mathbf{b}=\operatorname{col}(b, w)$. The combined input $\mathbf{u}=\operatorname{col}(u, w)$ drives an annihilation-only (passive) quantum system $G(s)$, as described in the previous section, to produce the output $\mathbf{y}=\operatorname{col}\left(y_{u}, y_{w}\right)$, although for filtering purposes, we are only interested in the output component $y_{u}$ which corresponds to the input channel $u$.

The objective: In the classical filtering theory [8], the equalizer is to compensate for signal distortions in the output $y_{u}(t)$, by minimizing the equalization error $e(t)=$ $\hat{u}(t)-u(t)$ between classical signals $\hat{u}(t), u(t)$ in the meansquare sense. The classical power spectrum density $P_{e, e}(i \omega)$ is usually $L_{2}$-integrable and is related to the correlation function of the error $e(t)$ via the inverse Fourier transform,

$$
R_{e, e}(t)=\frac{1}{2 \pi} \int_{-\infty}^{+\infty} P_{e, e}(i \omega) e^{i \omega t} d \omega .
$$

In this case, minimizing the mean-square error covariance measure $\operatorname{tr} R_{e, e}(0)$ is equivalent to minimizing $\operatorname{tr} P_{e, e}(i \omega)$ pointwise in $\omega$. Alternatively, the optimal causal filter can be sought to satisfy the Wiener-Hopf equation [8],

$$
R_{u, y_{u}}(t)=\int_{0}^{+\infty} h(t-\tau) R_{y_{u}, y_{u}}(\tau) d \tau, \quad t>0
$$

here $h(t)$ is the unilateral inverse Laplace transform of a causal transfer function $H(s)$. The equation (13) reflects the projection property of classical least-square estimates, $\mathbb{E}\left(e(t) y_{u}(\tau)^{\dagger}\right)=0$ for $-\infty<\tau<t$. The solution to equation (13) is obtained using spectral factorization.

Analogous to the classical mean-square equalization, we wish to obtain a quantum system $H(s)$ whose output $\hat{u}$ matches the input $u$ optimally, in the sense that the equalization error $e(t)=\hat{u}(t)-u(t)$ must have a minimum covariance. Owing to the physical realizability requirement reflected in the identity (7), quantum channels are not guaranteed to generate $L_{2}$-integrable power spectrum densities. For this reason, we will pose the problem directly as optimization of the power spectrum density, to either minimize $\operatorname{tr} P_{e, e}(i \omega)$ pointwise for every $\omega$, or obtain a causal $H(s)$ by solving the corresponding spectral factorization problem. Both approaches will be discussed in Section $\mathrm{V}$

Admissible equalizing filters: The key distinction of the problem under consideration from classical counterparts is that the system $H(s)$ must be physically realizable as a quantum system. This mandates imposing additional requirements on the filter. Firstly, to ensure that the LTI filter system obtained from the optimization problem 16 or from spectral factorization can be made physically realizable, it may need to be equipped with additional noise inputs $z-$ it was observed in [6], [14] that any LTI system can be made physically realizable by adding noise. Without loss of generality, we will assume that the added noise $z$ is in a Gaussian vacuum state, i.e., the corresponding mean and covariance of $z$ are

$$
\langle z(t)\rangle=0, \quad\left\langle\left[\begin{array}{c}
z(t) \\
z^{\#}(t)
\end{array}\right]\left[\begin{array}{c}
z^{\dagger}\left(t^{\prime}\right) \\
z^{T}\left(t^{\prime}\right)
\end{array}\right]\right\rangle=\left[\begin{array}{ll}
I & 0 \\
0 & 0
\end{array}\right] \delta\left(t-t^{\prime}\right)(.14)
$$

Secondly, to facilitate implementation of the resulting quantum filter [11], we restrict attention to passive equalizer systems. In this case, the requirement for physical realizability of the filter leads to a formal constraint of the form (7) on the transfer function $H(s)$ :

$$
H(s)\left[H\left(-s^{*}\right)\right]^{\dagger}=I
$$

Let us denote the set of passive physically realizable equalizers satisfying (15) as $\mathscr{H}_{r}$. The pointwise optimization of $\operatorname{tr} P_{e, e}(i \omega)$ in the class of physically realizable filters is thus a constrained optimization problem,

$$
\min _{H \in \mathscr{H}_{r}} \operatorname{tr} P_{e, e}(i \omega) \text {. }
$$

The constraint (15) precludes the direct application of standard filtering techniques to obtain an optimal quantum Wiener equalizer. In the next section we outline a relaxation technique which helps to overcome this problem.

\section{Constraint Relaxation}

Let us define the partitions of the transfer functions $G(s)$ and $H(s)$ compatible with the partitions of $\mathbf{u}=\operatorname{col}(u, w)$, $\mathbf{y}=\operatorname{col}\left(y_{u}, y_{w}\right)$, and $\operatorname{col}\left(y_{u}, z\right), \operatorname{col}(\hat{u}, \hat{z})$, respectively:

$$
G(s)=\left[\begin{array}{l}
G_{11}(s) G_{12}(s) \\
G_{21}(s) G_{22}(s)
\end{array}\right], \quad H(s)=\left[\begin{array}{l}
H_{11}(s) H_{12}(s) \\
H_{21}(s) H_{22}(s)
\end{array}\right] .
$$

With this notation, we have that

$$
\begin{aligned}
& P_{e, e}(s) \\
& =\left(H_{11}(s) G_{11}(s)-I\right)\left(I+\Sigma_{b}^{T}\right)\left(G_{11}\left(-s^{*}\right)^{\dagger} H_{11}\left(-s^{*}\right)^{\dagger}-I\right) \\
& +H_{11}(s) G_{12}(s)\left(I+\Sigma_{w}^{T}\right) G_{12}\left(-s^{*}\right)^{\dagger} H_{11}\left(-s^{*}\right)^{\dagger} \\
& +H_{12}(s) H_{12}\left(-s^{*}\right)^{\dagger} .
\end{aligned}
$$

Also, the constraint 15 is equivalent to

$$
\begin{aligned}
& H_{11}(s) H_{11}\left(-s^{*}\right)^{\dagger}+H_{12}(s) H_{12}\left(-s^{*}\right)^{\dagger}=I, \\
& H_{11}(s) H_{21}\left(-s^{*}\right)^{\dagger}+H_{12}(s) H_{22}\left(-s^{*}\right)^{\dagger}=0 \\
& H_{21}(s) H_{21}\left(-s^{*}\right)^{\dagger}+H_{22}(s) H_{22}\left(-s^{*}\right)^{\dagger}=I .
\end{aligned}
$$

From 18, we observe that the spectral density function $P_{e, e}(s)$ depends on the variables $H_{11}, H_{12}$ only. Therefore one possible approach to solving the equalizer design problem is to employ a two-step procedure whose first step is to optimize the equalization error with respect to $H_{11}(s)$, $H_{12}(s)$, subject to the constraint [19), followed by the second step during which the remaining transfer functions $H_{21}(s)$, $H_{22}(s)$ are computed to fulfill the remaining physical realizability constraints 20, 21.

Of course, there is no guarantee that with $H_{11}(s), H_{12}(s)$ found during the first step, the remaining transfer functions 
$H_{21}(s), H_{22}(s)$ exist and satisfy the conditions (20), 21]. Nevertheless, this approach is attractive in that it allows us to obtain tractable relaxations of the original quantum equalizer design problem. Indeed, using (19), $H_{12}(s)$ can be eliminated from the expression 18):

$$
\begin{aligned}
P_{e, e}(s)= & \left(H_{11}(s) G_{11}(s)-I\right)\left(I+\Sigma_{b}^{T}\right) \\
& \times\left(G_{11}\left(-s^{*}\right)^{\dagger} H_{11}\left(-s^{*}\right)^{\dagger}-I\right) \\
& +H_{11}(s) G_{12}(s)\left(I+\Sigma_{w}^{T}\right) G_{12}\left(-s^{*}\right)^{\dagger} H_{11}\left(-s^{*}\right)^{\dagger} \\
& -H_{11}(s) H_{11}\left(-s^{*}\right)^{\dagger}+I .
\end{aligned}
$$

It also follows from (19) that

$$
H_{11}(i \omega) H_{11}(i \omega)^{\dagger} \leq I \quad \forall \omega \in \mathbf{R}^{1} .
$$

This allows us to replace the original problem of finding an optimal passive equalizer $H(s)$ with the problem of optimizing the equalization error in the class of causal transfer functions $H_{11}(s)$ subject to the quadratic constraint (23). We will give a precise meaning to this statement in the next section, where we discuss two relaxed quantum Wiener filter problem formulations.

\section{TWO APPROACHES TO QUANTUM WIENER EQUALIZATION}

In this section we apply the relaxation technique discussed in the previous section to two problems which demonstrate features of the quantum Wiener filtering. Our aim is to highlight new features of the problem of coherent Wiener equalization owing to the physical realizability constraint (15), rather than obtain a general solution to this problem. All signals in this section are assumed to be scalar unless specified otherwise.

\section{A. Equalization via optimization of power spectrum density: An optical beam splitter}

In this section, we focus on the problem (16). The constraint relaxation proposed in the previous section allows to replace this problem with the problem involving the constraint 23). In the case of scalar signals $u, y_{u}$ and $\hat{u}, P_{e e}(s)$ and $\Sigma_{b}$ are scalars, and this problems simplifies significantly:

$$
\begin{aligned}
& \min _{\left|H_{11}(i \omega)\right| \leq 1} P_{e, e}(i \omega), \\
P_{e, e}(i \omega)= & \left(1+\Sigma_{b}\right)\left|H_{11}(i \omega) G_{11}(i \omega)-1\right|^{2} \\
& +\left|H_{11}(i \omega)\right|^{2} G_{12}(i \omega)\left(I+\Sigma_{w}^{T}\right) G_{12}(i \omega)^{\dagger} \\
& -\left|H_{11}(i \omega)\right|^{2}+1 .
\end{aligned}
$$

In (24), the minimum is taken over the set of causal transfer functions $H_{11}(s)$ subject to the scalar version of the condition (23). Obviously, we have in this case

$$
\min _{\left|H_{11}(i \omega)\right| \leq 1} P_{e, e}(i \omega) \leq \min _{H \in \mathscr{H}_{r}} P_{e, e}(i \omega) ;
$$

i.e., the problem (24) delivers a lower bound on the optimal power spectrum density. The requirement for $H_{11}(s)$ to be causal is also nontrivial - while the frequency pointwise optimization is easy to perform over complex $H_{11}$, the

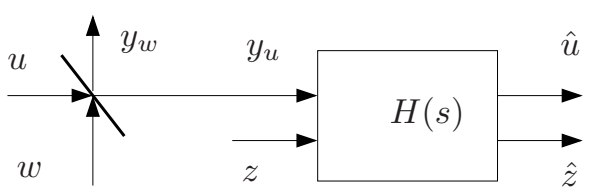

Fig. 2. A beam splitter and a quantum equalizer system.

pointwise optimal $H_{11, \omega}$ obtained this way must admit a causal extension into the complex plane. In general, this issue can be addressed numerically [1], using the standard Matlab software [10]. Therefore in the remainder of this section, we will be concerned with equalization of a static quantum system for which the causality condition is satisfied automatically. This simplified analysis aims to demonstrate that the proposed relaxation can lead to physically realizable equalizers which are optimal in the sense of (16).

As an example of a static quantum system consider a quantum-mechanical beam splitter, which is a two-input twooutput quantum system; see Fig. 2. In Fig. 2, the input $u$ represents the signal we would like to split, and the second input $w$ is an auxiliary noise input. The beam splitter mixes the signals $u$ and $w$, its outputs and inputs are related via a unitary transformation:

$$
\left[\begin{array}{l}
y_{u} \\
y_{w}
\end{array}\right]=G\left[\begin{array}{c}
u \\
w
\end{array}\right], \quad G(s)=\left[\begin{array}{cc}
\sqrt{\eta} & \sqrt{1-\eta} \\
-\sqrt{1-\eta} & \sqrt{\eta}
\end{array}\right] ;
$$

$\eta \in(0,1)$ is a real parameter known as transmittance. That is, $G(s)$ is static in this case, and

$$
y_{u}=\sqrt{\eta} u+\sqrt{1-\eta} w .
$$

The equalization problem is to estimate the signal $u$ from the output $y_{u}$ of this device using a coherent equalizer, i.e., a device which preserves the canonical commutation relations.

To demonstrate the application of a quantum Wiener filter in this problem, suppose that the input noise $b$ in (12) is in Gaussian vacuum state, and $\Sigma_{b}=0, \Pi_{b}=0$, whereas the beamsplitter noise $w$ is in a Gaussian thermal state, so that $\Sigma_{w}=\sigma_{w}^{2}>0, \Pi_{w}=0$. With these assumptions, the expression for the objective function in 25 becomes

$P_{e, e}(i \omega)=(1-\eta) \sigma_{w}^{2}\left|H_{11}(i \omega)\right|^{2}-2 \sqrt{\eta} \operatorname{Re} H_{11}(i \omega)+2 .(28)$

The constraint condition (23) reduces in this case to

$$
\left|H_{11}(i \omega)\right|^{2} \leq 1 \text {. }
$$

Since all coefficients in (28) are constants, the optimal value and the optimal equalizer should also be constant. The problem (24) is thus a regular constrained optimization problem, which can be solved using the Lagrange multiplier technique.

Proposition 1: 1. If $\sigma_{w}^{2} \leq \frac{\sqrt{\eta}}{(1-\eta)}$, then the optimal equalizer which attains minimum in (16) is $H(s)=I$.

2. On the other hand, when $\sigma_{w}^{2}>\frac{\sqrt{\eta}}{(1-\eta)}$, an optimal equalizer is given by

$$
\begin{aligned}
& H_{11}(s)=\frac{\sqrt{\eta}}{\sigma_{w}^{2}(1-\eta)}, \quad H_{12}(s)=\sqrt{1-\frac{\eta}{\sigma_{w}^{4}(1-\eta)^{2}}}, \\
& H_{21}(s)=-H_{12}(s), \quad H_{22}(s)=H_{11}(s) .
\end{aligned}
$$




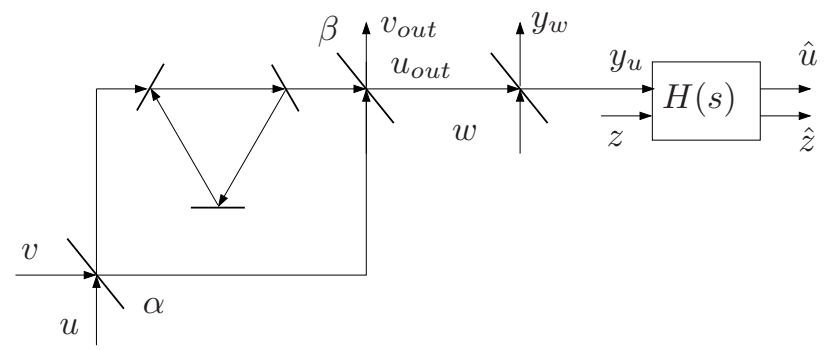

Fig. 3. A cavity, beam splitters and an equalizer system.

Such an equalizer attenuates the input $y_{u}$, and must include an additional noise input $z$, to ensure that it is physical realizable.

The corresponding expressions for the optimal error power spectrum density are

$$
\min _{H \in \mathscr{H}_{r}} P_{e e}= \begin{cases}\sigma_{w}^{2}(1-\eta)-2 \sqrt{\eta}+2, & \text { if } \sigma_{w}^{2} \leq \frac{\sqrt{\eta}}{(1-\eta)} \\ 2-\frac{\eta}{\sigma^{2}(1-\eta)}, & \text { if } \sigma_{w}^{2}>\frac{\sqrt{\eta}}{(1-\eta)}\end{cases}
$$

Comparing the power spectrum density of the error at the input of the filter, $P_{\left(y_{u}-u\right),\left(y_{u}-u\right)}(i \omega)$, with $P_{e, e}(i \omega)$ in [31, we observe that $P_{\left(y_{u}-u\right),\left(y_{u}-u\right)}(i \omega)=P_{e, e}(i \omega)$ if $\sigma_{w}^{2} \leq$ $\frac{\sqrt{\eta}}{(1-\eta)}$, and $P_{\left(y_{u}-u\right),\left(y_{u}-u\right)}(i \omega)>P_{e, e}(i \omega)$ if $\sigma_{w}^{2}>\frac{\sqrt{\eta}}{(1-\eta)}$. Thus, Proposition 1 shows that the requirement for physical realizability restricts the capacity of an optimal coherent equalizer to respond to noise in the input signal. It is still possible to reduce the MSE by means of a coherent equalizer, however this is only possible provided the covariance of the thermal noise in the input signal is sufficiently large. This situation differs strikingly from the classical Wiener equalization theory.

\section{B. The Wiener-Hopf technique for quantum equalization: An equalizer for an optical cavity}

Let us modify the system in Fig. 2 to include an optical cavity and two additional beam splitters of transmittance $\alpha$ and $\beta$; see Fig. 3. With these modification the system becomes dynamical. In Fig. 3. $v$ denotes an additional thermal Gaussian noise input into the system, with zero mean and covariance

$$
\left\langle\left[\begin{array}{c}
v(t) \\
v^{*}(t)
\end{array}\right]\left[\begin{array}{c}
v^{*}\left(t^{\prime}\right) \\
v\left(t^{\prime}\right)
\end{array}\right]\right\rangle=\left[\begin{array}{cc}
1+\sigma_{v}^{2} & 0 \\
0 & \sigma_{v}^{2}
\end{array}\right] \delta\left(t-t^{\prime}\right) .
$$

Correspondingly, the relation between the channel output $\operatorname{col}\left(y_{u}, y_{w}\right)$ and its input $\operatorname{col}(u, v)$ is found from the relations

$$
\begin{aligned}
{\left[\begin{array}{l}
y_{u} \\
y_{w}
\end{array}\right] } & =\left[\begin{array}{cc}
\sqrt{\eta} & \sqrt{1-\eta} \\
-\sqrt{1-\eta} & \sqrt{\eta}
\end{array}\right]\left[\begin{array}{c}
u_{\text {out }} \\
w
\end{array}\right], \\
{\left[\begin{array}{l}
u_{\text {out }} \\
v_{\text {out }}
\end{array}\right] } & =\bar{G}(s)\left[\begin{array}{l}
u \\
v
\end{array}\right], \\
\bar{G}(s) & =\left[\begin{array}{l}
\bar{G}_{11}(s) \bar{G}_{12}(s) \\
\bar{G}_{21}(s) \bar{G}_{22}(s)
\end{array}\right] \\
& =\sqrt{\alpha \beta}\left[\begin{array}{cc}
G_{c}-\sqrt{\alpha^{\prime} \beta^{\prime}} & \sqrt{\alpha^{\prime}} G_{c}+\sqrt{\beta^{\prime}} \\
-\sqrt{\beta^{\prime}} G_{c}-\sqrt{\alpha^{\prime}} & -\sqrt{\alpha^{\prime} \beta^{\prime}} G_{c}+1
\end{array}\right] ;
\end{aligned}
$$

$G_{c}(s)$ denotes the transfer function of the optical cavity

$$
G_{c}(s)=\frac{s-\frac{\gamma}{2}+i \Omega}{s+\frac{\gamma}{2}+i \Omega}
$$

$\gamma, \Omega$ are real constants, and $\alpha^{\prime}=\frac{1-\alpha}{\alpha}, \beta^{\prime}=\frac{1-\beta}{\beta}$. Note that $G_{c}(s)\left[G_{c}\left(-s^{*}\right)\right]^{*}=I$.

After these modifications, the power spectrum density of the equalization error in equation (22) is expressed as

$$
\begin{aligned}
P_{e, e}(s) & =2+\left(\eta \sigma_{v}^{2} \bar{G}_{12}(s)\left[\bar{G}_{12}\left(-s^{*}\right)\right]^{*}+(1-\eta) \sigma_{w}^{2}\right) \\
& \times H_{11}(s)\left[H_{11}\left(-s^{*}\right)\right]^{*} \\
& -\sqrt{\eta}\left(H_{11}(s) \bar{G}_{11}(s)+\left[H_{11}\left(-s^{*}\right)\right]^{*}\left[\bar{G}_{11}\left(-s^{*}\right)\right]^{*}\right) .
\end{aligned}
$$

The auxiliary optimization problem considered in the previous sections is therefore to obtain a causal transfer function $H_{11}(s)$ which optimizes (34) subject to the constraint 29.

Unlike the previous section, the system contains dynamics and the corresponding optimal filter is expected to be dynamical. Therefore, we cannot expect that the pointwise optimization in 24 will produce a causal transfer function $H_{11}(s)$. In the classical case, this issue is resolved using the Wiener-Hopf spectral factorization method [8]. Therefore, here we proceed as follows. First, we apply the Wiener-Hopf spectral factorization method [8] to obtain a causal optimal $H_{11}(s)$ that minimizes $\operatorname{tr} P_{e, e}(i \omega)$ for $P_{e, e}(s)$ in (34); this step does not involve the physical realizability constraints. Next, we show that in fact the found $H_{11}(s)$ validates the required constraint 29], provided the variance of the system noise exceeds a certain threshold. Then we show that in this case a complete physically realizable filter transfer function $H(s)$ which satisfies 19 21 can be constructed from the found $H_{11}(s)$.

Since $P_{e, e}(s)$ in 34 depends on $H_{11}(s)$ only, we can minimize $\operatorname{tr} P_{e, e}(i \omega)$ by treating $P_{e, e}(s)$ as a power spectrum density of a classical system. Define

$$
\zeta=\frac{1-\eta}{\eta} \frac{\sigma_{w}^{2}}{\alpha \beta}, \quad \rho=\frac{\alpha^{\prime}+\beta^{\prime}+\frac{\zeta}{\sigma_{v}^{2}}}{2 \sqrt{\alpha^{\prime} \beta^{\prime}}} \geq 1 .
$$

Letting $M(s)$ be the following causal transfer function,

$$
\begin{aligned}
M(s)= & \sqrt{2 \eta \sigma_{v}^{2} \sqrt{\alpha \beta(1-\alpha)(1-\beta)}(\rho+1)} \\
& \times \frac{s+\frac{\gamma}{2} \sqrt{\frac{\rho-1}{\rho+1}}+i \Omega}{s+\frac{\gamma}{2}+i \Omega},
\end{aligned}
$$

we obtain the identity

$$
M(s)\left[M\left(-s^{*}\right)\right]^{*}=\eta \sigma_{v}^{2} \bar{G}_{12}(s)\left[\bar{G}_{12}\left(-s^{*}\right)\right]^{*}+(1-\eta) \sigma_{w}^{2} .
$$

Therefore,

$$
\begin{aligned}
P_{e, e}(s)= & \left(\left(M(s) H_{11}(s)-\sqrt{\eta} Q(s)\right)\right. \\
& \times\left(\left(M(-s)^{*} H_{11}(-s)^{*}-\sqrt{\eta}\left[Q\left(-s^{*}\right)\right]^{*}\right)\right. \\
& -\eta Q(s)\left[Q\left(-s^{*}\right)\right]^{*}+2,
\end{aligned}
$$


where

$$
\begin{aligned}
& Q(s) \triangleq\left[\frac{\bar{G}_{11}\left(-s^{*}\right)}{M\left(-s^{*}\right)}\right]^{*} \\
& =\frac{1-\sqrt{\alpha^{\prime} \beta^{\prime}}}{\sqrt{2 \eta \sigma_{v}^{2} \sqrt{\alpha^{\prime} \beta^{\prime}}(\rho+1)}}\left(1+\frac{\frac{\gamma}{2}\left(\sqrt{\frac{\rho-1}{\rho+1}}+\frac{1+\sqrt{\alpha^{\prime} \beta^{\prime}}}{1-\sqrt{\alpha^{\prime} \beta^{\prime}}}\right)}{s+i \Omega-\frac{\gamma}{2} \sqrt{\frac{\rho-1}{\rho+1}}}(\supsetneqq 8 .)\right.
\end{aligned}
$$

Now consider a classical filtering problem of minimizing the MSE between the filter output $\hat{u}=H(s) y_{u}$, where $y_{u}=M(s) u$, and the signal $\bar{u}=\sqrt{\eta} Q(s) u$. Let $[Q(s)]_{+}$ denote the causal part of $Q(s)$. According to the WienerHopf method [8], the causal solution to this problem is

$$
H_{11}(s)=\frac{\sqrt{\eta}}{M(s)}[Q(s)]_{+} .
$$

This filter ensures that the error $u-\bar{u}$ and the filter input $y_{u}$ are orthogonal. Since the expression for the power spectrum density of the error in this problem is exactly equal to the first term in (37), we conclude that the filter 39 minimizes $P_{e, e}(i \omega)$ in the class of causal transfer functions. This yields the explicit expression for the optimal filter which is causal by way of construction:

$$
H_{11}(s)=\frac{\left(1-\sqrt{\alpha^{\prime} \beta^{\prime}}\right) / \sqrt{\eta}}{\sigma_{v}^{2}\left(\sqrt{\alpha^{\prime}}+\sqrt{\beta^{\prime}}\right)^{2}+\zeta} \times \frac{s+\frac{\gamma}{2}+i \Omega}{s+\frac{\gamma}{2} \sqrt{\frac{\rho-1}{\rho+1}}+i \Omega} .
$$

Proposition 2: Under the condition

$$
\sigma_{v}^{2}>\frac{-\zeta\left(\alpha^{\prime}+\beta^{\prime}\right)+\sqrt{4 \zeta^{2} \alpha^{\prime} \beta^{\prime}+\frac{\left(1-\sqrt{\alpha^{\prime} \beta^{\prime}}\right)^{2}}{\eta \alpha \beta}\left(\alpha^{\prime}-\beta^{\prime}\right)^{2}}}{\left(\alpha^{\prime}-\beta^{\prime}\right)^{2}}
$$

the transfer function $H_{11}(s)$ in (40) satisfies 29).

It can be shown using Proposition 2 that the following constants are real under (41):

$$
\begin{aligned}
& \alpha_{11}=\frac{1-\sqrt{\alpha^{\prime} \beta^{\prime}}}{2 \sqrt{\eta} \sigma_{v}^{2}(\rho+1) \sqrt{(1-\alpha)(1-\beta)}}, \\
& \alpha_{12}=\sqrt{1-\alpha_{11}^{2}}, \quad \beta_{12}=\frac{\gamma}{2} \sqrt{\frac{\rho-1}{\rho+1}-\alpha_{11}^{2}} .
\end{aligned}
$$

Proposition 3: Suppose (41) holds. Then the optimal causal equalizer for the system under consideration in this section is given by the following transfer functions

$$
\begin{aligned}
& H_{11}(s)=\alpha_{11} \frac{s+\frac{\gamma}{2}+i \Omega}{s+\frac{\gamma}{2} \sqrt{\frac{\rho-1}{\rho+1}}+i \Omega}, \\
& H_{12}(s)=\frac{\alpha_{12} s+\beta_{12}+i \alpha_{12} \Omega}{s+\frac{\gamma}{2} \sqrt{\frac{\rho-1}{\rho+1}}+i \Omega}, \\
& H_{21}(s)=-\frac{\alpha_{12} s-\beta_{12}+i \alpha_{12} \Omega}{s+\frac{\gamma}{2} \sqrt{\frac{\rho-1}{\rho+1}}+i \Omega}, \\
& H_{22}(s)=\alpha_{11} \frac{s-\frac{\gamma}{2}+i \Omega}{s+\frac{\gamma}{2} \sqrt{\frac{\rho-1}{\rho+1}}+i \Omega} .
\end{aligned}
$$

As we see, the condition (41) plays a critical role in the above analysis. The expression on the right-hand side of (41) depends on $\sigma_{w}^{2}$. If

$$
\zeta\left(\alpha^{\prime}+\beta^{\prime}\right)>\sqrt{4 \zeta^{2} \alpha^{\prime} \beta^{\prime}+\frac{\left(1-\sqrt{\alpha^{\prime} \beta^{\prime}}\right)^{2}}{\eta \alpha \beta}\left(\alpha^{\prime}-\beta^{\prime}\right)^{2}},
$$

then this expression is negative, and 41 holds trivially. It can be shown that if $\sigma_{w}^{2}>\frac{\left|1-\sqrt{\alpha^{\prime} \beta^{\prime}}\right|}{\sqrt{1-\eta}}$ then (46) holds, and hence (41) is trivially satisfied. Thus we have arrived at a conclusion similar to that made in the previous section: If the variance of the thermal noise in the system is sufficiently large, then there exists a filter which attenuates the thermal noise component of $y_{u}$ while injecting a small amount of noise through the $z$ channel.

\section{CONCLUSIONS}

The paper has discussed a quantum counterpart of the classical Wiener filtering problem for equalization of quantum systems. The requirement to obtain a physically realizable passive causal equalizer imposes nonconvex constraints on the filter transfer function. We have discussed one form of relaxation of these constraints, and have shown, via examples, that the relaxation does not preclude finding a physically realizable coherent filter able to reduce the signal distortion caused by the noisy quantum channel.

\section{REFERENCES}

[1] Z. Drmac, S. Gugercin, and C. Beattie. Quadrature-based vector fitting for discretized $\mathscr{H}_{2}$ approximation. SIAM Journal on Scientific Computing, 37(2):A625-A652, 2015.

[2] J. E. Gough, M. R. James, and H. I. Nurdin. Squeezing components in linear quantum feedback networks. Physical Review A, 81(2):023804, 2010.

[3] J. E. Gough and G. Zhang. On realization theory of quantum linear systems. Automatica, 59:139-151, 2015.

[4] F. Grosshans and P. Grangier. Continuous variable quantum cryptography using coherent states. Physical review letters, 88(5):057902, 2002

[5] R. L. Hudson and K. R. Parthasarathy. Quantum ito's formula and stochastic evolutions. Communications in Mathematical Physics, 93(3):301-323, 1984

[6] M. R. James, H. I. Nurdin, and I. R. Petersen. $H^{\infty}$ control of linear quantum stochastic systems. IEEE Transactions on Automatic Control, 53(8):1787-1803, 2008.

[7] M.R. James and J.E. Gough. Quantum dissipative systems and feedback control design by interconnection. IEEE Transactions on Automatic Control, 55(8):1806 -1821, 2010.

[8] T. Kailath. Lectures on Wiener and Kalman filtering. Springer, 1981.

[9] A. I. Maalouf and I. R. Petersen. Bounded real properties for a class of annihilation-operator linear quantum systems. IEEE Transactions on Automatic Control, 56(4):786-801, 2011.

[10] Matlab. Tfest: Transfer function estimation. https://www.mathworks.com/help/ident/ref/tfest.html.

[11] H. I. Nurdin. On synthesis of linear quantum stochastic systems by pure cascading. IEEE Transactions on Automatic Control, 55(10):2439-2444, 2010.

[12] K. R. Parthasarathy. An introduction to quantum stochastic calculus. Birkhäuser, 2012.

[13] A. J. Shaiju and I. R. Petersen. A frequency domain condition for the physical realizability of linear quantum systems. IEEE Transactions on Automatic Control, 57(8):2033-2044, 2012.

[14] S. L. Vuglar and I. R. Petersen. How many quantum noises need to be added to make an LTI system physically realizable? In 2011 Australian Control Conference, pages 363-367, 2011.

[15] N. Wiener. The extrapolation, interpolation, and smoothing of stationary time series. Wiley, New York, 1949.

[16] G. Zhang and M. R James. On the response of quantum linear systems to single photon input fields. IEEE Transactions on Automatic Control, 58(5):1221-1235, 2013. 